is voiced in the tanguage of one of the ablest descriptive writers on astronomical subjects of the present day. On p. 127 of her late admirable work entitled "Problems in Astrophysics," Miss Clerke dismisses the subject with the words, "The only genuine 'eclipse comet' so far captured was that seen and photographed at Sohag I7th May, I882." This talented writer makes not the slightest reference to the fact that the 1893 phenomenon differed in one important particular from all those it is said to resemble-that it possessed the one observed element which even the genuine object does not lay claim to, namely, the photographs prove that this object moved, receded from the sun through an angular distance equal to two-thirds of the solar diameter in less than four hours. ${ }^{1}$ On the Mina Broncis photographs this object is plainly connected with the sun by a single, straight, isolated coronal stream. ${ }^{2}$

Ann Arbor, December 19, 1903.

\section{Dynamical and Granular Medid.}

I SHOULD be very much obliged if any reader of NATURE who has studied the matter could enlighten me on the following point.

We may regard a dynamical system as commonly understood as being a system which. when left to itself. obeys the Hamiltonian equations

$$
\frac{d x}{d t}=\frac{\partial \mathrm{U}}{\partial \xi}, \& c_{\cdot}, \quad \frac{\partial \xi}{\partial t}=-\frac{\partial \mathrm{U}}{\partial x},
$$

where $x, y, \ldots$. are generalised coordinates, $\xi, \eta \ldots$. . generalised momenta, and

$$
\mathrm{U}=\frac{1}{2}(\xi \xi) \xi^{2}+(\xi \eta) \xi \eta+\ldots+\mathrm{V}
$$

an $1 \mathrm{~V},(\xi \xi),(\xi \eta), \& \mathrm{c}$, , are any functions whatever of $x, y, \ldots$

We may regard a granular medium as a particular kind of system coming under this heading for which $U$ takes the form

$$
\left.\mathrm{U}=\Sigma_{r} \frac{\xi_{r}^{2}+\eta_{r}^{2}+\zeta_{r}^{2}}{2 m_{r}}+\Sigma \Sigma_{r s} \mathrm{~F}_{r s}\left\{\left[\left(x_{r}-x_{s}\right)^{2}+\left(y_{r}-y_{s}\right)^{2}+\left(z_{r}-z_{s}\right)^{2}\right]\right]^{2}\right\}(2)
$$

where $m_{r}$ stands for any constant (being the mass of the $r$ th particle, atom, corpuscle, grain, or whatever else you like to call it) and $F_{r s}$ is any function whatever, continuous or discontinuous, determined by the law of force between different masses.

What I want to know is this :--

(I) Is every dynamical system which can exist in Euclidean three-dimensional space transformable into a granular system according to the above definition by a proper choice of coordinates?

(2) If not, what are the precise mathematical conditions under which a dynamical system can be so transformed?

We may put these questions in a somewhat different form. It is undoubtedly possible to conceive a universe the physical phenomena of which are represented by equations of any assumed form whatever, and therefore not necessarily by the equations of dynamics. Is it possible to conceive a universe the physical phenomena of which are represented by dynamical equations, but cannot be accounted for by means of a granular medium? I have read many treatises and essays dealing with theories of the ether, in which it has been tacitly assumed that the only possible answer to (I) must inevitably be "yes," and I cannot but feel that a discussion in your columns might be of much use to physicists.

G. H. Bryan.

\section{Phosphorescence of Photographic Plates.}

I OBTAINED the following results, which are new to me, in the course of some experiments on the action of light on the salts of silver.

I have not yet thoroughly examined the light or radiation emitted in these experiments, but its actinic power is low, and it appears to render the brush discharge from an induction coil more luminous.

The sensitive silver salts, such as the bromide, iodide and chloride, if precipitated and kept in the dark, have the property, under certain conditions, of emitting light in degrees proportionate to their sensitiveness. Thus the

1 See Astronomical foumal, No. $3^{18}$. Also Mr. Wesley's article in

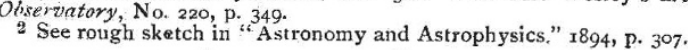
NO. I 785 , voL. 69$]$ bromide, which is the most sensitive, emits more light than the iodide and chloride. A convenient way of observing the phenomenon is to take a bromide photographic plate and place it at once (without having exposed it) in ordinary pyro soda developing solution and allow it to remain for ten minutes. Take out of the solution, wash, extinguish the "red lamp," and in total darkness plunge it suddenly into a dish containing a saturated solution of aluminium sulphate. The plate immediately becomes phosphorescent, and the solution also is luminous, but not so bright as the plate is at first. The light gradually weakens, and in a minute or two dies away. On pouring the solution off the plate into a bottle, the whole body of the liquid becomes luminous, and has the appearance of " bottled moonlight." It remains so several minutes, and the light is increased by shaking the liquid.

If half the plate be exposed to the action of white light for a second before treating with the pyro soda solution, that half remains dark and emits no light when the plate is put into the aluminium sulphate. If the plate is given a short exposure in the camera, and developed and put into the aluminium sulphate solution, the image will appear dark on a phosphorescent background.

On placing some precipitated bromide of silver (which had been kept a few days in a corked test-tube in the dark) in a porcelain dish and exposing it to a bright red light whilst adding the pyro-soda solution, it appears black, but on pouring off the solution the precipitate gradually assumes a bright green appearance under the red light, whilst in white light it appears dark grey or black.

The remarkable part of these experiments appears to me to be the fact that the exposing of the silver salts to the action of light destroys their power of emitting it under the treatment described, whilst the salt precipitated and treated in total darkness emits light freely.

\section{Ley Hill House, Sutton Coldfield.}

\section{T. A. Vacghton.}

\section{Formation of Coal}

SoME of your readers will no doubt be interested to see the photograph sent herewith, which represents a peculiar growth of coal on a piece of timber.

The timber is part of a wooden trough into which, for a period of three years, water had been delivered by tanks lifting the water from a coal-mine shaft.

The formation of coal was found adhering to the vertical sides of the trough, forming a miniature coal seam about a quarter of an inch thick. This coal is hard and bright, and its texture and solidity differ in no respect from ordinary coal.

The explanation seems to be that the water contained small quantities of fine coal dust abraded from the seam
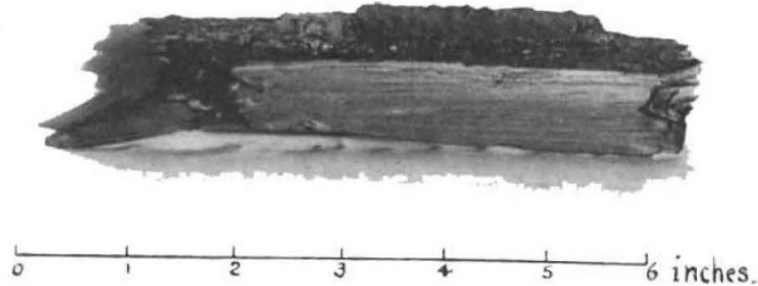

below, and these, either through the motion of the water or by some other means, were filtered out and formed anew into solid coal.

I believe this phenomenon has never previously been observed, and it appears to show that a coal seam may be broken up, washed away, and again built up in a new position withnut the aid of either the passage of time, pressure or heat.

Rainhill, January 3 .

HeNRY HaLl.

\section{The Lamprey.}

I SHALL feel obliged to any of your readers who will kindly tell me where to procure specimens of the lamprey. They are unobtainable at the Marine Stations of Millport and Plymouth. J. Pentland-Smith. St. Regulus, Park Place. Elie, N.B., January 5. 